\title{
MarkerCount: A stable, count-based cell type identifier for single cell RNA-Seq experiments
}

\begin{abstract}
Cell type identification is a key step to downstream analysis of single cell RNA-seq experiments. Indispensible information for this is gene expression, which is used to cluster cells, train the model and set rejection thresholds. Problem is they are subject to batch effect arising from different platforms and preprocessing. We present MarkerCount, which uses the number of markers expressed regardless of their expression level to initially identify cell types and, then, reassign cell type in clusterbasis. MarkerCount works both in reference and marker-based mode, where the latter utilizes only the existing lists of markers, while the former required pre-annotated dataset to train the model. The performance was evaluated and compared with the existing identifiers, both marker and referencebased, that can be customized with publicly available datasets and marker DB. The results show that MarkerCount provides a stable performance when comparing with other reference-based and markerbased cell type identifiers.
\end{abstract}

\section{Introduction}

Single-cell RNA-Seq technology[1-3] enabled transcriptomic analysis of micro environment in heterogeneous tissues [4], such as tumor micro environment. A key step to its analysis is the cell type identification, for which there are manual annotation tools, such as Seurat[5], SC3[6], SCANPY[7] and Alona[8], by which cell type annotations were made publicly available for further research. Automatic annotation pipelines were also developed, one of which is marker-based approach. This approach utilizes lists of markers to identify cell types using gene expression profile obtained from single cell RNA-Seq experiments. Garnett [9], SCINA[10], scSorter[11] and CellAssign[12] fall into this class, for which, there are several utilizable databases for cell type markers such as Panglao DB[13] and CellMarker DB[14]. Another approach is reference-based methods that utilize the existing annotation to obtain cell-type profiles to be used for identification, e.g., SingleR[15], scPred[16], scmap[17], CaSTLe[18] and CHETAH[19]. Various machine learning techniques, such as classification and clustering, were used as key enabling tools for such types of cell type identification.

In this work, we present MarkerCount, a count-base cell type identifier that support both markerbased and reference-based identification. The overall procedure is shown in Figure 1 and the detailed description can be found in the online method section. Briefly speaking, the data processing pipeline consists of two steps, (1) selecting markers for given reference and (2) identification of cell type utilizing "marker counts" and cluster-wise cell type correction, where the second step can be slightly modified to make it marker-based identification utilizing the existing cell type markers, e.g., those in [13] and [14]. The key is to suitably set rejection threshold to determine 'unknown' cell type, for example, 
unknown tumor cells, that is not in the set of target cell types. Most of the identifiers, either marker based or reference based, showed reasonable performance when there are no unknowns. However, a good identifier must be able to successfully identify such cell clusters for further study on their genomic characteristics not identified so far.

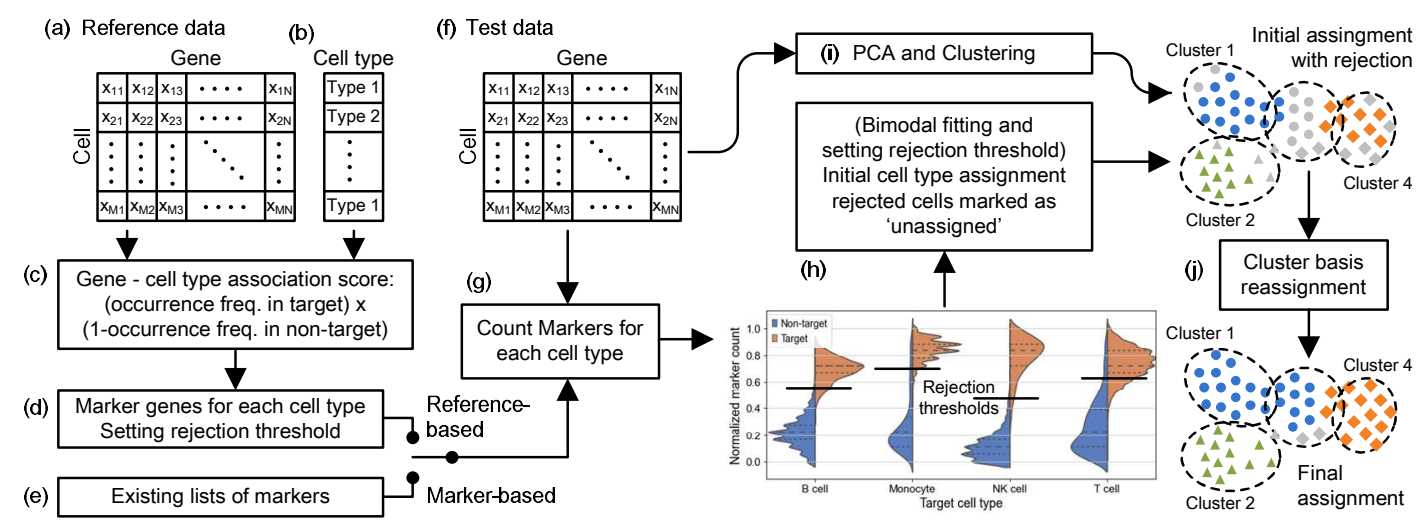

Figure 1 An overview of MarkerCount data processing. MarkerCount operates in both reference-based and marker-based mode. In the former, it requires reference data (a) along with cell type annotation (b) to find makers of each cell type (d), for which gene-cell type association score (c) is used. In markerbased mode, it uses the existing markers (e). In test phase, it take cell type markers and the test data (f) as input. Follwing data processing includes marker count (g), initial cell type assignment and rejection of unclear cells (h), PCA and clustering (i) and the cluster basis reassignment for final identification (j).

\section{Results}

For evaluation, we ran MarkerCount in both reference-based and marker-based mode and compared them separately with the existing reference-based identifiers [15, 17-19] and the markerbased ones [9-11] with the cell type markers in both [13] and [14], respectively. To this end, we used 7 single cell RNA-Seq data for which manual annotation is available. Among them, two are the Pancreas data reported in [20] (Pancreas 2K), [21] (Pancreas 8K), two are peripheral blood [22] (CBMC 8K) and [23] (PBMC 68K), consisting of various immune related cells, two are lung data used in [24] and [25], which can be downloaded from the human cell atlas (https://data.humancellatlas.org/) and the remaining two are from the tissue with tumor [26] (Melanoma 5K) and [27] (Head and Neck 6K). A summary on these datasets is shown in Supplemental Table 1. There are many other software packages for cell type annotation, such as Moana [28], DigitalCellSorter[29], Cell-BLAST[30]. However, they do not provide functions to build custom models either utilizing list of markers or reference data with annotation. Therefore, we consider only those with which one can build customized model for cell type identification.

In the reference-based identification, we considered four sets (Pancreas $2 \mathrm{~K}$ and Pancreas 8K), (CBMC 8K and PBMC 68K), (Lung 29K and Lung 39K) (Melanoma 5K, HeadNeck 6K) separately. For each of 
these, we performed cross-validation, i.e., circularly used one as test data and others as reference to identify marker genes and to set rejection threshold. To show the stable performance, we also tested tumor data using CBMC $8 \mathrm{~K}$ and/or PBMC $68 \mathrm{~K}$ as reference to find markers for various immune related cells including T cells, B cells, monocytes, dendritic cells and so on. Noting that no tumor cells were annotated in CBMC 8K while there are many tumor cells in Melanoma and HeadNeck data. those tumor cells and the cell not annotated in the reference must be ideally determined as 'unknown'.

Figure 2 (a) to (c) summarize the results, where, similar to those in [19], we considered 5 criteria, that is, correct $(\mathrm{C})$, error(E), erroneously assigned (EA), erroneously unassigned (EUA) and correctly unassigned (CUA). These are defined according to (1) whether any label assigned by the identification or not (i.e., marked as unknown, unclear or unassigned), (2) whether the assigned label is in the reference or not and (3) if it is in the reference cell type, whether the predicted label is the same as the assigned label or not. The detailed definitions are described in the online method section.

Ideally, only C and CUA should exist, which was depicted in the first bar in all figures. Imperfect identification causes E, EA and/or EUA, each of which may have different impact according to the purpose of the analysis. Although too much EUA is undesired, E and EA might have worse impact than EUA in most cases as one can manually characterizes clusters predicted as unknown or unassigned. Roughly speaking, it is desirable that the predicted results is as close to the ideal case (the first bar) as possible. In pancreas and blood data, there is very small portion of cells of which their type is not in the references while, the melanoma and head-neck data contain a large portion of tumor cells that must be identified as unknown (or unassigned). For the pancreas data, SingleR has shown to be the best in terms of the correct prediction and MarkerCount, scmap(cell), CaSTLe, CHETAH, scmap (cluster) follow. However, if E and EA are desired as small as possible, scmap(cell and cluster) or CHETAH look better than SingleR, MarkerCount and CaSTLe, even though scmap(cluster) and CHETAH unassigned cell type for large portion of cells. In blood data, MarkerCount showed best performance in terms of correct prediction. Although scamp (cell) and scmap (cluster) showed very small error, their EUAs are too large and the correct precision are very small. The prediction accuracy in terms of CUA is highlighted in Figure 1 (c), where MarkerCount showed closest pattern to ideal case with small portion of $\mathrm{E}$ and EA. Other cell type identifiers, such as CHETAH, scmap (cell) and scmap (cluster), showed smaller E than MarkerCount and they erroneously assigned tumor cells to other types of normal cell type. Overall, the MarkerCount showed better performance than others, especially, for the blood and tumor datasets. Although its performance for pancreas was not the best, it performed reasonably good.

To show the performance of MarkerCount, we performed experiments on the tumor datasets using blood datasets as references in different configuration. Figure 1 (d) to (e) show the performance of tumor data, respectively, with CBMC 8K, PBMC 68K and both as references. In all three setups, the performances of MarkerCount were not only the best but also almost the same, while other referencebased identifiers were more variant than MarkerCount is. 
In Supplemental Figure 1, we plotted Sankey diagram between the manual annotation and the predicted one for blood and tumor data, respectively. The figure shows that the cell types having sufficient number of cells shows better performance than those with smaller number of cells. Having large number of cells, they can be well clustered and well characterized so that one can better identify those cell types while the identification model for those cell types with small number of cells tends to be overfitted to the data. Doubtlessly, we need more cells to improve the identification performance. The problem is that uneven cell population is inherent. One may combine two or more dataset into one reference data to train the model to finally improve the performance. However, it may not be always true because of batch effect. Most of the cell type identifiers utilizes clustering algorithm, anyhow, and the identification model is fitted in cluster basis. Problem arises if there are two or more clusters far apart for one cell type. Another issue to consider is the cell types that are highly differentiable, such as monocytes, macrophages and dendritic cells. As shown in Supplemental Figure 1 and 2, some of these cell types were hardly recovered and interchangeably detected. In many cases, they are not well clustered with sufficient separation as clearly shown in Supplemental Figure 2.

Unlike other reference-based methods, MarkerCount provides also marker-based identification with a slight modification. Therefore, we also compared MarkerCount with the existing marker-based methods, including Garnett [9], SCINA [10] and scSorter [11] (We couldn't run CellAssign [12] due to installation error). Marker-based approach requires only lists of cell markers for which we used CellMarker DB [14]. We extracted cell markers for pancreas, Lung, blood and peripheral blood, where the last two were used for CBMC 8K, PBMC 68K, Melanoma 5K and HeadNeck 6K. The results are summarized in Figure 2 for the four datasets, pancreas, peripheral blood (CBMC and PBMC), lung and tumor tissues. Similar to the comparison of reference-based cell type identifiers, we measured 5 criteria, C, EUA, E, EA and CUA. For pancreas dataset, scSorter performed slightly better than MarkerCount. However, for all other datasets, MarkerCount showed the best performance.

The performance of marker-based identifiers depends necessarily on the set of markers used and the selection of good markers is crucial to obtain a good result. One reason seems to be the uneven number of cell markers. In CellMarker DB, several subtypes of dendritic cells have hundreds of marker genes while others, e.g., natural killer cells and B cells, have only around ten markers. Such uneven number of markers may degrade the identification performance and this is why MarkerCount (marker-based mode) reselect markers if too many markers is provided in marker DB (see online method). Depending on the specific procedures and algorithm of cell type identifiers, the best set of markers will also be different. Literally, 'cell marker' means that it is expressed only in a specific cell type and not expressed at all elsewhere. However, it seems not be always hold since the definition of cell type can be quite different according to the analytical purposes. For example, one can required only identify $\mathrm{T}$ cells as a whole in some applications, while one may require identification of specific subtypes, such as cytotoxic $\mathrm{T}$ cells, memory T cells, helper T cells and regulatory $\mathrm{T}$ cells. Depending on what specific set of cell 
types are required, different approach and/or different tools must be used. Supplemental Figure 3 and 4 show the Sankey diagram and the UMAP plots similar to those in the reference-based identification experiments. In Supplemental Figure 3 and 4 , we see that specific subtypes are interchangeably identified to other subtypes within the same broader cell types. Having markers of both broad and specific cell type, simultaneous identification of broad cell types and specific cell types may cause unexpected results as shown in Supplemental Figure 3. As it is also risky to identify specific cell type directly using only a few markers, a better option is to use hierarchical identification, i.e., identify broad cell type first using many markers and then determine their subtypes using a few specific markers. What specific hierarchy should be used depends on specific tissues to analyze.

(a) Pancreas_2K,Pancreas_8K cross-validated

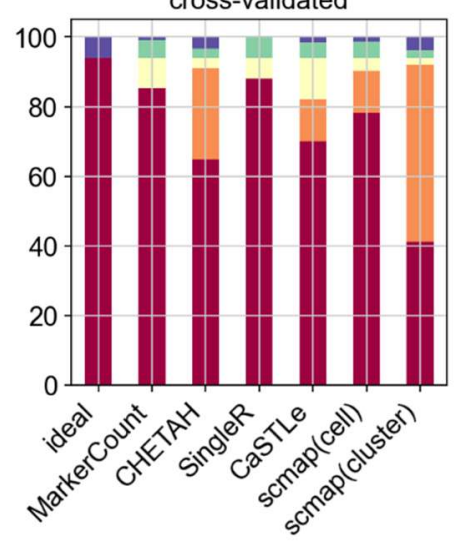

Method

(d) HeadNeck_6K,Melanoma_5K cross-validated

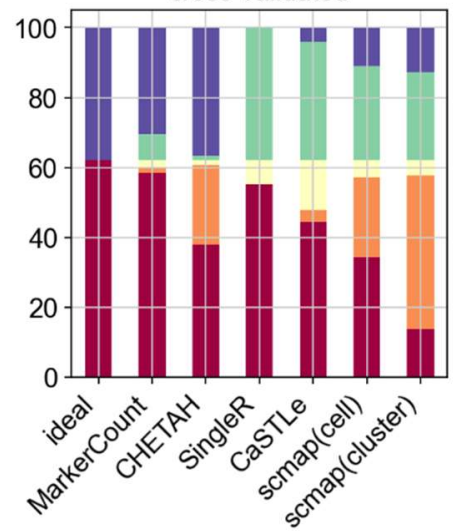

Method (b) CBMC_8K,PBMC_68K cross-validated

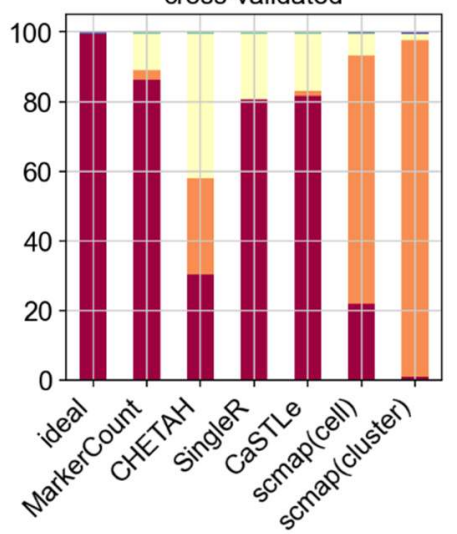

Method

(e) HeadNeck_6K,Melanoma_5K with $\mathrm{CBMC}-8 \mathrm{~K}$ as reference

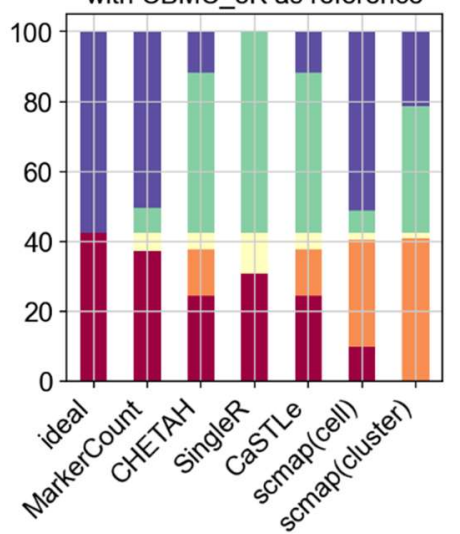

Method (c) Lung_29K,Lung_39K cross-validated

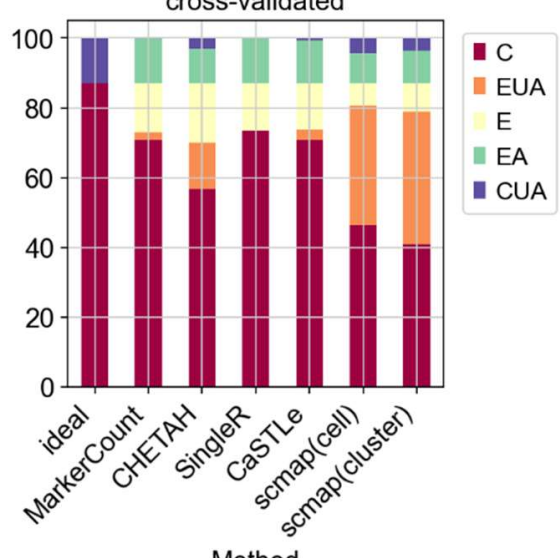

Method

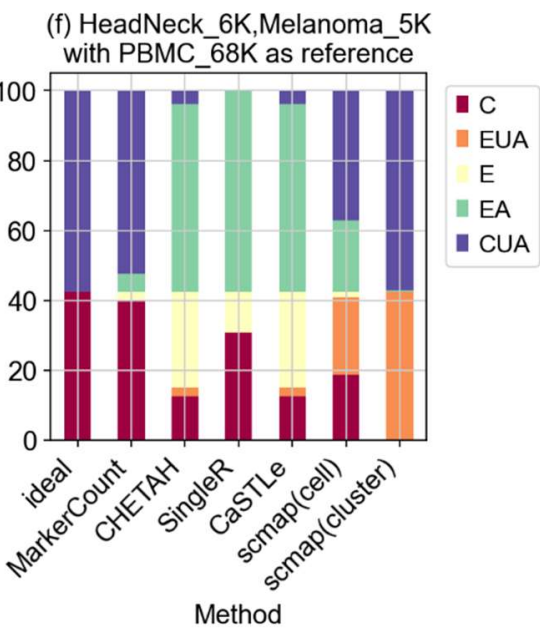

Figure 2 Comparisons of the reference-based cell type identification performances of MarkerCount, CHETAH, SingleR, CaSTLe, scmap (cell) and scmap (cluster). (a) Pancreas data, (b) Peripheral Blood (immune system) and (c) Tissues with tumor. C: Correct, EUA: Erroneously unassigned, E: Error, EA: Erroneously assigned, CUA: Correctly unassigned. The first bar in each figure shows the ideal performance, where CUA account for tumor cells and those cell types not in the reference. Ideally, they must be predicted as unknown (or unassigned). 


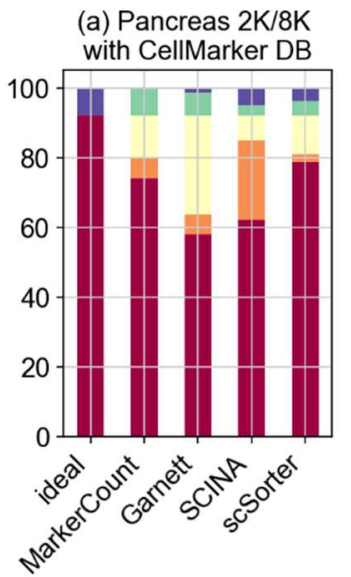

Method

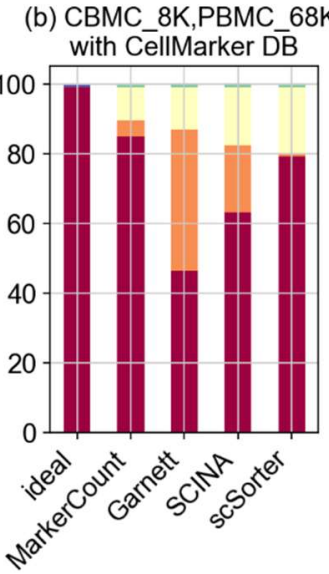

Method

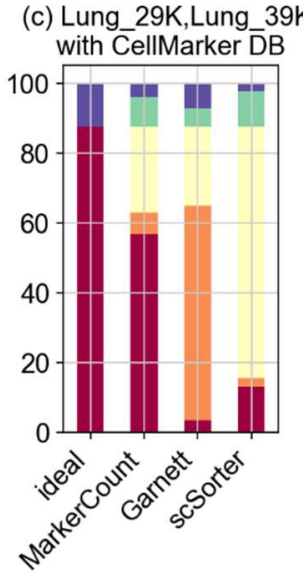

Method

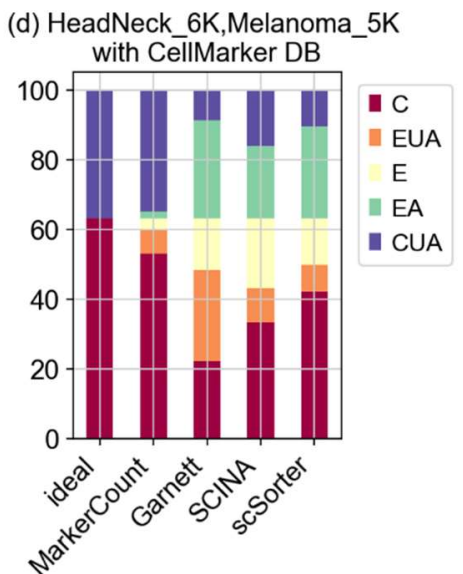

Method

Figure 3 Comparisons of the marker-based cell type identification performances of MarkerCount, scSorter, Garnett, and SCINA. (a) Pancreas data, (b) Peripheral Blood (immune system), (c) Lung and (d) Tissues with tumor, with CellMarker DB markers. C: Correct, EUA: Erroneously unassigned, E: Error, EA: Erroneously assigned, CUA: Correctly unassigned. The first bar in each figure shows the ideal performance, where CUA account for tumor cells and those cell types not in the reference cell type. Ideally, they must be predicted as unknown (or unassigned).

\section{Discussion}

Marker-based approach and reference-based approach have their own advantage and disadvantage. In both approaches, one of key components is clustering and, for a good identification, each cell types should be well clustered, which is not always the case. Although many clustering algorithms, such as partitioning-based, distribution-based or graph-based, are available, performance difference with different clustering seems not critical. Rather, handling unclear clusters of highly differentiable cells, as in monocytes, macrophage and dendritic cells, seems to be more important issue. The marker-based approach does not require reference annotation, which is a big advantage compared to reference-based approach. However, it depends highly on the selection of markers and the best set of markers should be selected taking the specific identification procedures into account. Reference-based approaches select their own markers internally and look more robust if the reference annotation is sufficiently reliable. However, it is subject to batch effect when working with datasets from different platforms and preprocessing and using the binary indication of gene expression, as in MarkerCount, makes it more robust to the batch effect.

\section{Method}

The operation of MarkerCount can be divided into two steps, (1) Finding markers for each cell types using reference data and (2) Use markers to identify cell types in cluster basis.

\section{Finding markers}


Using the prior annotation provided with the gene expression matrix, reference cell types are identified first. Let $G$ be the set of genes and $x_{i j}$ be the genes expression of the $i$ th cell and $j$ th gene. We first convert them to binary indicator $b_{i j} \in\{0,1\}$ representing whether the gene is expressed in that cell or not, i.e., $b_{i j}=1$ if $x_{i j}>0$ or 0 otherwise. We then compute marker score $s_{m, j}$ for a cell type $m$ as

$$
s_{m, j}=\left(\frac{1}{\left|C_{m}\right|} \sum_{i \in C_{m}} b_{i j}\right)\left(1-\frac{1}{\left|\bar{C}_{m}\right|} \sum_{i \in \bar{C}_{m}} b_{i j}\right)^{n}
$$

where $C_{m}$ is the set of cells annotated as of type $m$ and $\bar{C}_{m}$ is the complementary set of $C_{m} .\left|C_{m}\right|$ is the cardinality of $C_{m} \cdot \frac{1}{\left|C_{m}\right|} \sum_{i \in C_{m}} b_{i j}$ is simply the occurrence frequency of the $j$ th gene in the $m$ th cell type. $n$ is an hyperparameter relatively weighing the second term. We set it 2 in all the experiments performed in this work. Then, for each cell type $m$, we sort $s_{m, j}$ in descending order and select first $N$ genes as its markers. $N$ is also an hyper-parameter and we fixed it to 18 for all the cell types in this work. Note that the selection of marker genes is done separately for each cell type and it is possible for two or more cell types to share some common markers. In addition, before comparing the score in (1), one can narrow down the candidate genes by enforcing the condition, $\frac{1}{\left|C_{m}\right|} \sum_{i \in C_{m}} b_{i j} \geq f_{t h}$ for some occurrence frequency threshold $f_{t h}$ which we set 0.9 .

\section{Marker count}

In test phase, for given binary expression profile of the $i$ th cell, $b_{i j}$, we obtain the normalized marker count $y_{i, m}=\frac{1}{N} \sum_{j \in M_{m}} b_{i j}$ where $M_{m}$ is the set of markers of the $m$ th cell type. The cell type is initially determined by taking the maximum of $y_{i, m}$, i.e., $m_{i}^{*}=\underset{m}{\operatorname{argmax}} y_{i, m}$, which is accepted if $\underset{m}{\max } y_{m}$ is greater than or equal to the cell type specific rejection threshold, $t_{m}$. Otherwise, the cell is marked as 'unassigned'.

\section{Obtaining the rejection threshold}

The count threshold $t_{m}$ can be obtain in various ways. In this work, we considered two approach, i.e., parametric and non-parametric. In non-parametric approach, we directly obtain from $y_{m}{ }^{\prime}$ s. Consider two set of cells for given rejection threshold $t, C_{m}(t)$ and $C_{m}^{*}(t)$. The former is the set of cells (in the reference data) of which the true (manually annotated) cell type is $m$ and its normalized marker count $y_{i, m} \geq t$, while the latter is the set of cells that are decided to be the $m$ th cell type according to $y_{i, m}$ satisfying (1) $y_{i, m}>y_{i, m}$ for all other cell types $m^{\prime}$ and (2) $y_{i, m} \geq t$. Then, the false positive rate can be defined as

$$
F P R(t)=1-\frac{\left|C_{m}(t) \cap c_{m}^{*}(t)\right|}{\left|C_{m}(t)\right|}
$$

where the second term is the true positive rate. The objective is to find $t$ such that $F P R(t) \approx p$ for given target $F P R, p$. In non-parametric approach, one can find $t$ by sorting $y_{i, m}$ in descending order 
and find minimum $y_{i, m}$ such that $F P R(t) \leq p$. In parametric approach, we use univariate Gaussian mixture model with two components, i.e., $y_{i, m} \sim N\left(\mu_{1}, \sigma_{1}^{2}\right)$ for $m \in C_{m}$ and $y_{i, m} \sim N\left(\mu_{0}, \sigma_{0}^{2}\right)$ for $m^{\prime} \in$ $\bar{C}_{m}$. Denoting the relative size of $C_{m}$ and $\bar{C}_{m}$ as $\pi_{0}$ and $\pi_{1}$, respectively, $F P R(t)$ can be defined as

$$
F P R(t)=\frac{F_{0}(t)}{F_{1}(t)+F_{0}(t)} \text { with } F_{k}(t) \equiv \int_{t}^{\infty} \pi_{k} N\left(y ; \mu_{k}, \sigma_{k}^{2}\right) d y
$$

The parametric approach especially useful for marker-based operation of MarkerCount, where reference data is not available. Given test data only, one can resort to the bimodal fitting, for which expectation maximization (EM) algorithm can be used to find $\pi_{k}, \mu_{k}, \sigma_{k}^{2}$ for $k=0,1$. Note that SCINA also used bimodal fitting. But, it applied to gene expression level, while MarkerCount applies it to the normalized marker counts $y_{i, m}$ to determine the rejection threshold. In reference-based mode, the rejection thresholds for all the cell types are computed in the training phase using the reference data using either (2) or (3), while, in marker-based mode, it can be obtained by applying bimodal fitting to the test data. Supplemental Figure 5 shows comparisons of the two distributions of $C_{m}$ and $\bar{C}_{m}$ for some cell types in pancreas, CBMC 8K and PBMC68K.

\section{Cluster basis cell type reassignment}

Although marker count based cell type identification works reasonably well, one can improve the identification accuracy, specifically the recall, by employing cluster basis reassignment. The procedure is as follows. We first perform clustering. While some clusters have all its cells assigned by a cell type, others may not be fully covered or not covered at all. The latter might be unknown cells that does not exist in the reference cell type. However, the former may be supposed to be a specific cell type that was not fully covered due to high rejection threshold. We can reassign cell types to those unassigned cells in a partially covered region in cluster by cluster fashion. To this end, we first identify cell types that are partially occupying the cluster. With their centroids, covariance matrices and size, we reassign a cell type to those unassigned by comparing their distances from the centroids as follows.

$$
d\left(\mathbf{z}, \boldsymbol{\mu}_{m} ; \boldsymbol{\Sigma}_{m}, \pi_{m}\right)=\frac{1}{\pi_{m}}\left(\mathbf{z}-\boldsymbol{\mu}_{m}\right)^{T}\left(\boldsymbol{\Sigma}_{m}+\rho \boldsymbol{I}\right)^{-1}\left(\mathbf{z}-\boldsymbol{\mu}_{m}\right)
$$

where $\mathbf{z}$ is a dimension-reduced gene expression profile of a cell, $\boldsymbol{\mu}_{m}, \boldsymbol{\Sigma}_{m}, \pi_{m}$ are the centroid (mean), covariance matrix and the relative size of the $m$ th cell type that reside in the cluster and $\rho$ is a regularization constant, which we set 0.1 of the average variances. By comparing the weighted Mahalanobis distance in (4) of an unassigned cell from the centroids for the cell types in that cluster, we decide its cell type to the closest one. For the dimension reduction, we applied principal component analysis (PCA) as typically used in the preprocessing for clustering. The reassignment is performed if the portion of the unassigned cells is below a certain threshold, say 0.8. Although more sophisticated methods can be devised, we used this rather simple heuristic approach. 


\section{Reference-based and marker-based operation of MarkerCount}

Using the functions described above, the operation of MarkerCount can now be described more succinctly. In the reference-based operation, MarkerCount uses the reference data and its annotation to find the best markers for each cell type in the reference. Using these markers, it computes the normalized marker counts and decide initial prediction of cell types for all the cells in the reference data. Using the initial prediction and the annotated cell type, the rejection thresholds are obtained. The outputs of the training phase consist of the sets of markers and the rejection threshold for each cell type, which are then used for cell type identification for the test data. In the test phase, we first assign cell types to those cells whose normalized marker count is above the rejection threshold. Then clustering is performed for the test data and the distance-based reassignment is applied in cluster by cluster basis. In marker-based mode, on the other hand, we first compute the normalized marker counts for all the cells and all the cell types utilizing the existing markers. For each cell type, bimodal fitting is applied to determine the rejection threshold for each cell type. One possible problem in the marker-based mode is the uneven number of markers. In this case, cell types with a few markers tend to get higher priority than those with much larger number of markers. This does not happen in the reference-based mode as we select the same or at least similar number of markers for each cell type. To handle this problem, we applied the following tricks.

\section{Resolving uneven number of markers}

One possible solution is to reselect markers for those cell types with large number of markers. That is, in the first round of cell type assignment, we assign cell types with higher rejection threshold and use the prediction in the first around to obtain the score in (1) for all the markers and finally reselect markers that have higher scores. This is done only for those cell types that has the number of markers larger than the desired numbers. This approach, however, solve the problem only partially since there exist cell types having only a few or sometimes only one specific markers. To resolve this problem, we applied penalty weight per cell type according to their number of markers as $w=\left(1+3 e^{-(n-1) / 2}\right)^{-1}$, where $n$ is the number of markers. The penalty weight $w$ is multiplied to the normalized marker count before obtaining the rejection threshold and making cell type assignment. Although these tricks were devised for the case when the existing marker DB is used, the best way to avoid such problem in MarkerCount is to use the same or similar number of markers for each cell type, in which case, the penalty weight will be the same for all the cell types so that it does not affect the MarkerCount operation.

\section{Dataset and cell type renaming}

To properly evaluate and compare performances, we collected 6 single cell RNA-Seq data with manual annotation available. Those data were used in [19] and partially in other works. The description of the data is summarized in Supplemental Table 1. In all data, we did not take into count those cells annotated 
as 'unknown' or 'unclear' in the performance evaluation even though we performed identification anyway.

Two tumor data and CBMC 8K used rather broad cell types, such as T cells, NK cells and dendritic cells, while PBMC 68K used specific types, such as CD8+/CD45RA+ Naive Cytotoxic, CD4+ T Helper2, CD4+/CD45RA+/CD25- Naive T, CD8+ Cytotoxic T, CD4+/CD45RO+ Memory and CD4+/CD25 T Reg. Therefore, in the comparison of reference-based identifiers, we renamed PBMC cell types to their corresponding broad cell type, e.g., Naive Cytotoxic, T Helper2, Naive T, Cytotoxic T, Memory T and T Reg were all mapped to one broad type, $\mathrm{T}$ cell, to train the models for all the reference-based identifier. In marker-based, the two marker DB provide markers for specific cell type along with those for broad cell type. For example, CellMarker DB provides $87 \mathrm{~T}$ cell markers along with several specific markers

for CD4+, CD8+, CD4+ memory T cells, and so on. Therefore, we used the markers as is for identification of specific cell type. The problem was that, when identifying specific cell types, the performances were unacceptable for all the identifiers we examined, as shown in Supplemental Figure 3. Hence, in the comparison of marker-based identifiers, we renamed after identification, using the predicted specific cell types. As a matter of fact, immune related cells are highly differentiable so that they do not form clearly separable clusters as shown in Supplemental Figure 2 and 4. Moreover, it is questionable that the manual annotation can be used as ground truth since it is typically done in cluster basis, which limits the resolution of cell type clustering.

\section{Performance criteria}

Five performance measures, C, E, EA, CUA and EUA, are defined as follows:

A. C is the portion of cells that has an assigned label, one from the reference cell type, and the predicted label is the same as the original assignment.

B. E is the portion of cells that has an assigned label existing in the reference, while the predicted label is different from the original one.

C. EA is those cells that has an assigned label but that is not in the reference and the identifier predicted as one of the reference cell type

D. CUA is those that has an assigned label but that is not in the reference, including tumor cells, and the identifier predicted as unknown (or unassigned).

E. EUA is those that has an assigned label in the reference and the identifier predicted as unknown (or unassigned).

In general, there exists tradeoff between the two. Although it is also informative to show precision versus recall, we used these more specific measures to give better insight into the performance of cell type identifiers.

\section{Cross validation for the reference-based identification}

To show the effectiveness of the reference-based cell type identification, we used cross validation, 
where, with $M$ datasets, one is selected for test while others are used as reference to find cell type markers and this is repeated by circularly shifting their role in the experiment. This was done for the results in Figure 1 (a) to (d). For the results in Figure 1 (e) and (f), the reference and the test data were fixed.

\section{References}

1. Tang, F. C., Barbacioru, C., Wang, Y. Z., Nordman, E., Lee, C., Xu, N. L., et al. (2009). mRNA-Seq whole-transcriptome analysis of a single cell. Nat. Methods 6, 377-386. doi: 10.1038/nmeth.1315

2. Picelli, S., Bjorklund, A. K., Faridani, O. R., Sagasser, S., Winberg, G., and Sandberg, R. (2013). Smart-seq2 for sensitive full-length transcriptome profiling in single cells. Nat. Methods 10, 10961098. doi: 10.1038/nmeth.2639

3. Kolodziejczyk, A. A., Kim, J. K., Svensson, V., Marioni, J. C., and Teichmann, S. A. (2015). The technology and biology of single-cell RNA sequencing. Mol. Cell 58, 610-620. doi: 10.1016/j.molcel.2015.04.005

4. Chung, W., Eum, H. H., Lee, H. O., Lee, K. M., Lee, H. B., Kim, K. T., et al. (2017). Single-cell RNAseq enables comprehensive tumour and immune cell profiling in primary breast cancer. Nat. Commun. 8:15081. doi: 10.1038/ncomms15081

5. Satija, Rahul, et al. Spatial reconstruction of single-cell gene expression data. Nature biotechnology 2015;33:495-502.

6. Kiselev, Vladimir Yu, et al. SC3: consensus clustering of single-cell RNA-seq data. Nature methods 2017;14:483-486.

7. Wolf, F. Alexander, Philipp Angerer, and Fabian J. Theis. SCANPY: large-scale single-cell gene expression data analysis. Genome biology 2018;19:1-5.

8. Franzén, Oscar, and Johan LM Björkegren. alona: a web server for single-cell RNA-seq analysis. Bioinformatics 2020;36:3910-3912.

9. Pliner, Hannah A., Jay Shendure, and Cole Trapnell. "Supervised classification enables rapid annotation of cell atlases." Nature methods 2019;16:983-986.

10. Zhang, Ze, et al. SCINA: a semi-supervised subtyping algorithm of single cells and bulk samples. Genes 2019;10:531.

11. Guo, Hongyu, and Jun Li. scSorter: assigning cells to known cell types according to marker genes. Genome biology 2021;22: 1-18.

12. Campbell, K. R., S. P. Shah, and A. W. Zhang. Assigning scRNA-seq data to known and de novo cell types using CellAssign. 2019;2019

13. Franzén, Oscar, Li-Ming Gan, and Johan LM Björkegren. PanglaoDB: a web server for exploration of mouse and human single-cell RNA sequencing data. Database 2019;2019

14. Zhang, Xinxin, et al. CellMarker: a manually curated resource of cell markers in human and mouse. Nucleic acids research 2019;47:D721-D728. 
15. Aran, Dvir, et al. Reference-based analysis of lung single-cell sequencing reveals a transitional profibrotic macrophage. Nature immunology 2019;20:163-172.

16. Alquicira-Hernandez, Jose, et al. scPred: accurate supervised method for cell-type classification from single-cell RNA-seq data. Genome biology 2019;20:1-17.

17. Kiselev, Vladimir Yu, Andrew Yiu, and Martin Hemberg. scmap: projection of single-cell RNA-seq data across data sets. Nature methods 2018;15:359-362.

18. Lieberman, Yuval, Lior Rokach, and Tal Shay. CaSTLe-classification of single cells by transfer learning: harnessing the power of publicly available single cell RNA sequencing experiments to annotate new experiments. PloS one 2018;13:e0205499.

19. de Kanter, Jurrian K., et al. CHETAH: a selective, hierarchical cell type identification method for single-cell RNA sequencing. Nucleic acids research 2019;47:e95-e95.

20. Muraro, Mauro J., et al. A single-cell transcriptome atlas of the human pancreas. Cell systems 2016;3:385-394.

21. Baron, Maayan, et al. A single-cell transcriptomic map of the human and mouse pancreas reveals inter-and intra-cell population structure. Cell systems 2016;3:346-360.

22. Stoeckius, Marlon, et al. Simultaneous epitope and transcriptome measurement in single cells. Nature methods 2017;14:865-868.

23. Zheng, Grace $X Y$, et al. Massively parallel digital transcriptional profiling of single cells. Nature communications 2017;8:1-12.

24. Madissoon, E., Wilbrey-Clark, A., Miragaia, R.J. et al. scRNA-seq assessment of the human lung, spleen, and esophagus tissue stability after cold preservation. Genome Biol 21, 1 (2020)

25. Reyfman PA, Walter JM, Joshi N, et al. Single-Cell Transcriptomic Analysis of Human Lung Provides Insights into the Pathobiology of Pulmonary Fibrosis. Am J Respir Crit Care Med. 2019;199(12):1517-1536.

26. Tirosh, Itay, et al. Dissecting the multicellular ecosystem of metastatic melanoma by single-cell RNA-seq. Science 2016;352:189-196.

27. Puram, Sidharth V., et al. Single-cell transcriptomic analysis of primary and metastatic tumor ecosystems in head and neck cancer. Cell 2017;171:1611-1624.

28. Wagner, Florian, and Itai Yanai. Moana: A robust and scalable cell type classification framework for single-cell RNA-Seq data. BioRxiv 2018;456129.

29. Domanskyi, Sergii, et al. Polled Digital Cell Sorter (p-DCS): Automatic identification of hematological cell types from single cell RNA-sequencing clusters. BMC bioinformatics 2019;20:116.

30. Cao, Zhi-Jie, et al. Searching large-scale scRNA-seq databases via unbiased cell embedding with Cell BLAST. Nature communications 2020;11:1-13. 


\section{Supplemental Tables and Figures}

Supplemental Table 1. A summary of datasets used for the analysis

\begin{tabular}{|c|c|c|c|c|}
\hline Data & Protocol & Num. of cells total & $\begin{array}{c}\text { Num. of } \\
\text { Tumor cells }\end{array}$ & $\begin{array}{c}\text { Num of cell } \\
\text { types }\end{array}$ \\
\hline Pancreas 2K & inDrops & 2126 & None & $10(10)$ \\
\hline Pancreas 8K & CEL-seq2 & 8569 & None & $14(13)$ \\
\hline CBMC 8K & Drop-seq & 8617 & None & $15(11)$ \\
\hline PBMC 68K & $10 X$ genomics & 68579 & None & $11(6)$ \\
\hline Lung 29K & $10 X$ v2 & 57202 down sampled by 1/2 & None & $28(18)$ \\
\hline Lung 38K & $10 X$ v2 & 114396 down sampled by 1/3 & None & $31(24)$ \\
\hline Melanoma 5K & Smart-seq2 & 4513 & 1251 & $11(8)$ \\
\hline HeadNeck 6K & Smart-seq2 & 5902 & 2215 & $10(10)$ \\
\hline
\end{tabular}

These data can be downloaded from the following sites.

Pancreas 2K: https://www.ncbi.nlm.nih.gov/geo/query/acc.cgi?acc=GSE84133

Pancreas 8K: https://www.ncbi.nlm.nih.gov/geo/query/acc.cgi?acc=GSE85241.

CBMC 8K: https://www.ncbi.nlm.nih.gov/geo/query/acc.cgi?acc=GSE100866.

PBMC 68K: https://www.ncbi.nlm.nih.gov/geo/query/acc.cgi?acc=GSE93421.

Lung 29K: https://data.humancellatlas.org/explore/projects/c4077b3c-5c98-4d26-a614-246d12c2e5d7

Lung 39K: https://data.humancellatlas.org/explore/projects/c1a9a93d-d9de-4e65-9619-a9cec1052eaa

Melanoma 5K https://www.ncbi.nlm.nih.gov/geo/query/acc.cgi?acc=GSE72056.

HeadNeck 6K https://www.ncbi.nlm.nih.gov/geo/query/acc.cgi?acc=GSE103322. 
(a) Blood - MarkerCount

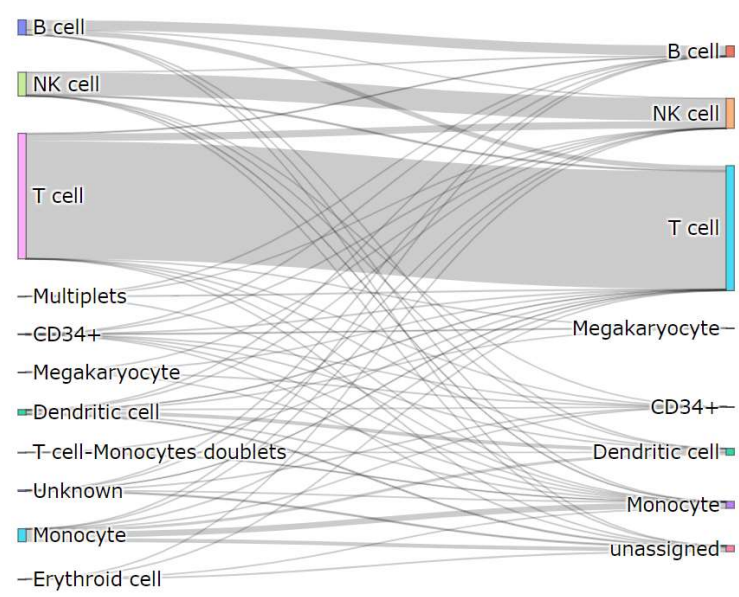

(c) Tumor - MarkerCount
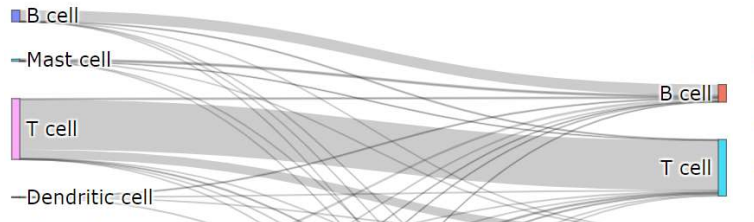

-NK-cell NKicellI

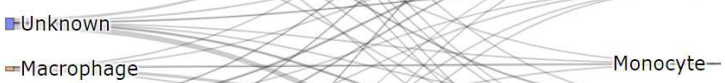

-Macrophage Monocyte-
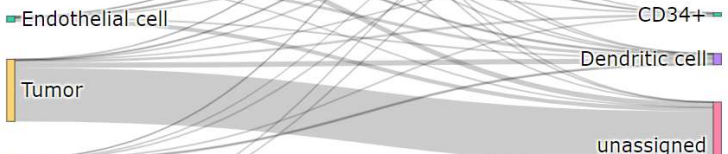

Fibroblast

-Myocyte- (b) Blood - CaSTLe

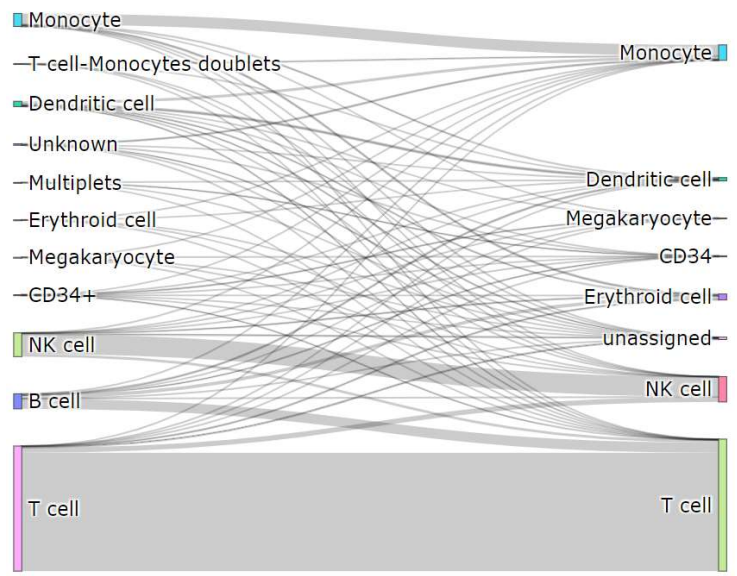

(d) Tumor - CHETAH

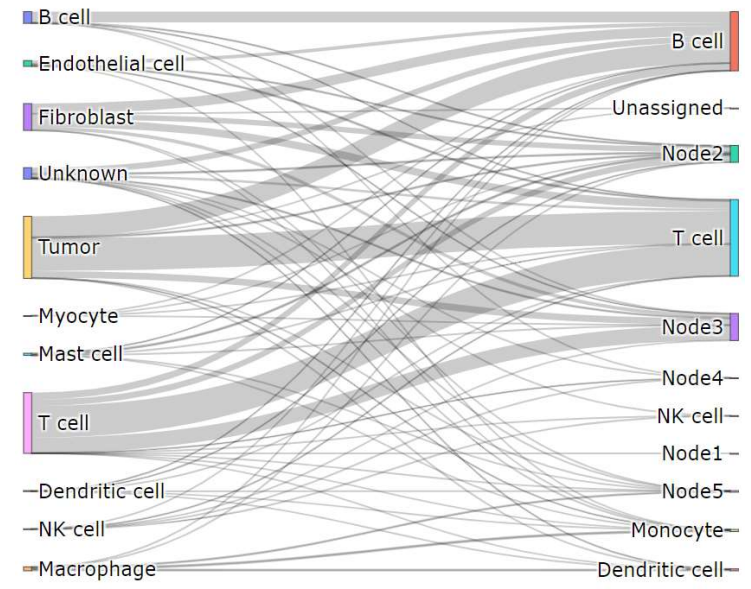

Supplemental Figure 1. Sankey diagram for two datasets with different reference-based identifiers (two best identifiers for each data). (a) blood data with MarkerCount, (b) blood data with CaSTLe, (c) tumor with MarkerCount and (d) tumor with CHETAH. We used broad cell type for PBMC 68K as the other provide only broad cell type. Those cell types with limited number of cells tend to get more errors than those with larger number of cells. 

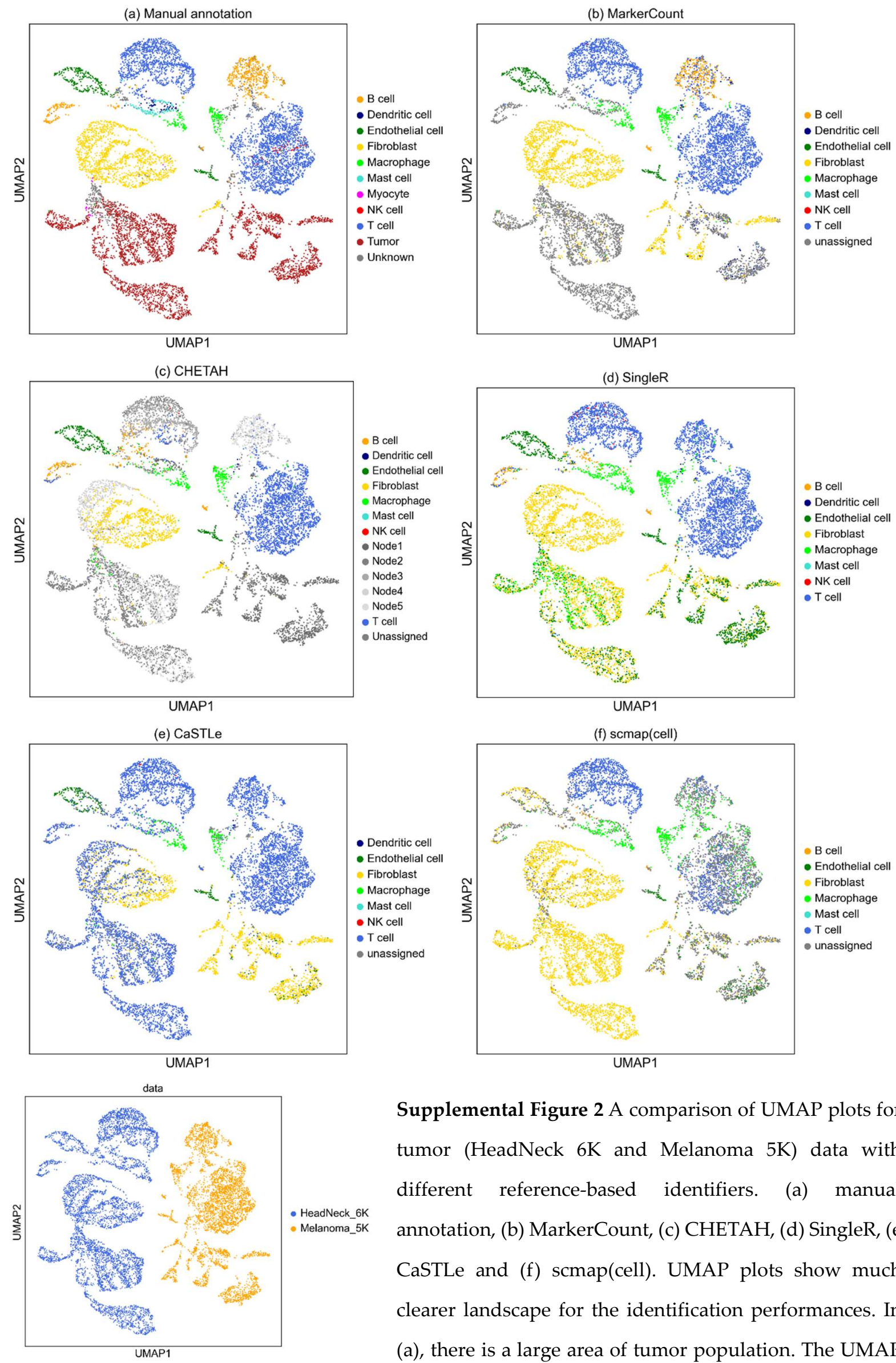

Supplemental Figure 2 A comparison of UMAP plots for tumor (HeadNeck 6K and Melanoma 5K) data with different reference-based identifiers. (a) manual annotation, (b) MarkerCount, (c) CHETAH, (d) SingleR, (e) CaSTLe and (f) scmap(cell). UMAP plots show much clearer landscape for the identification performances. In (a), there is a large area of tumor population. The UMAP plots were computed using dimension reduced version of gene expression matrix. 
(a) Tumor - MarkerCount

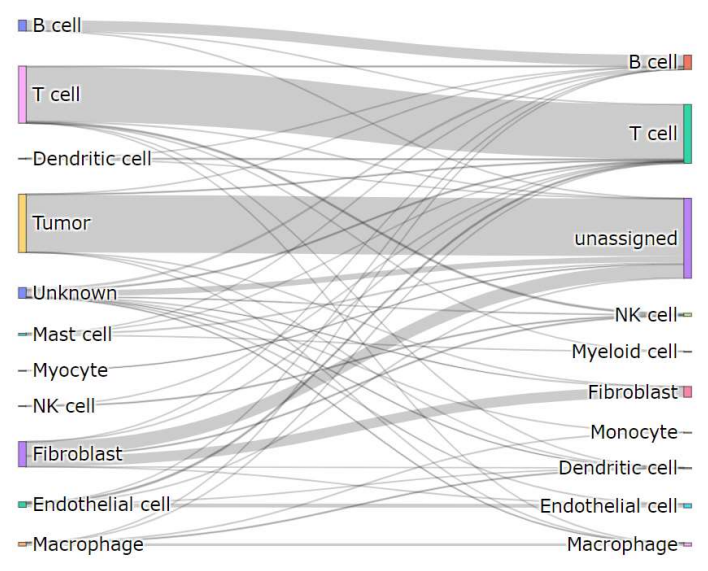

(c) Blood - MarkerCount

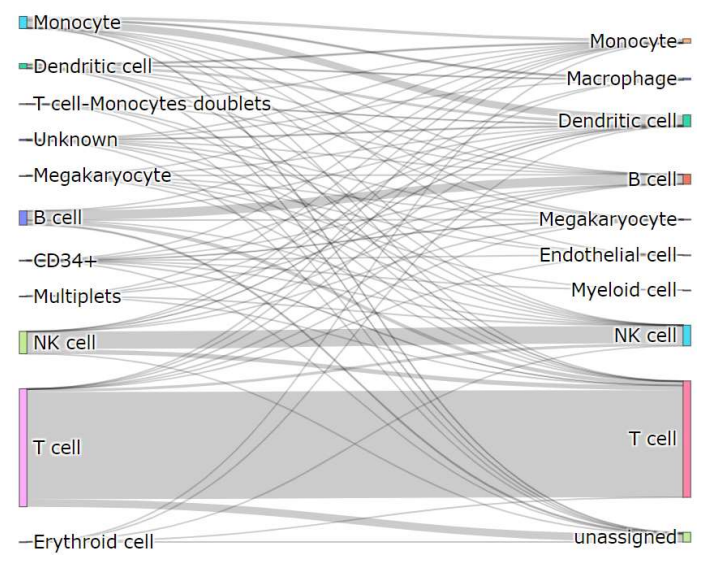

(e) PBMC 68K (Specific cell type) - MarkerCount

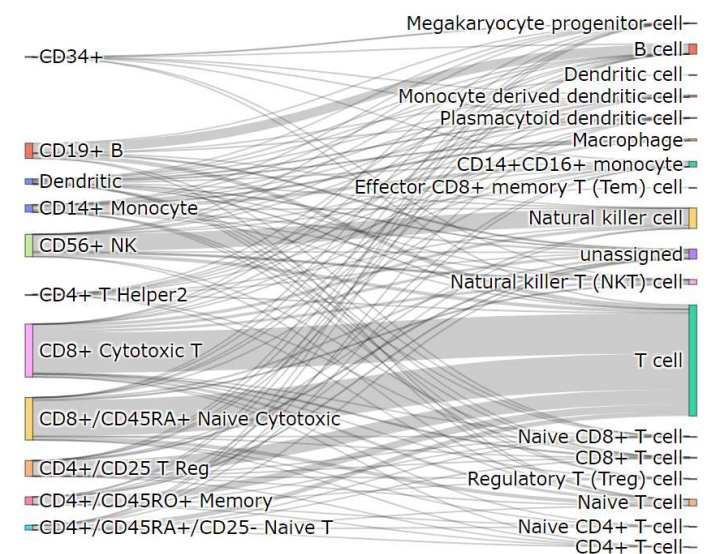

(b) Tumor - SCINA

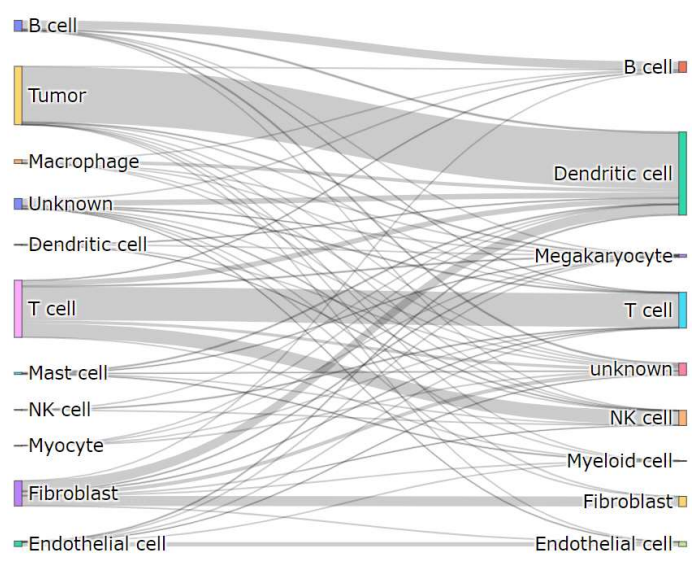

(d) Blood - Garnett

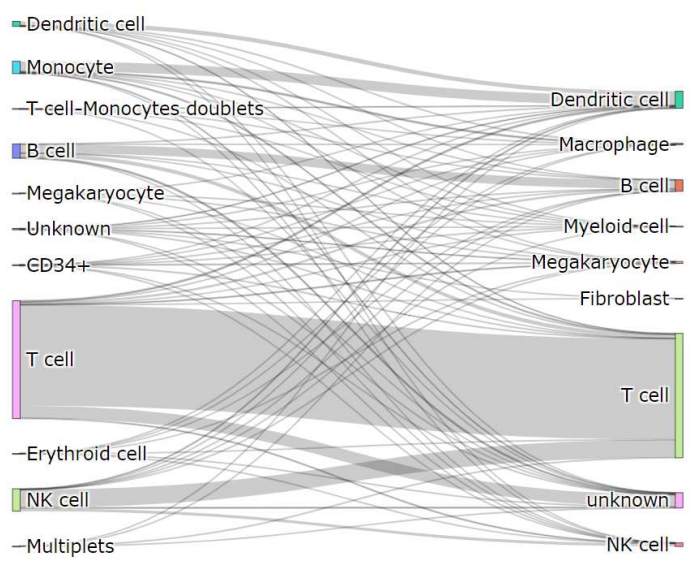

(f) PBMC 68K (Specific cell type) - Garnett

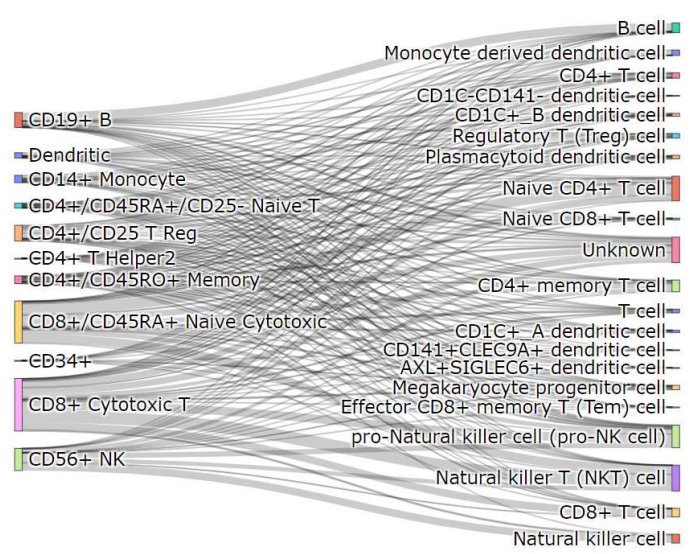

Supplemental Figure 3. Sankey diagram for tumor and blood datasets with different marker-based identifiers (two best identifiers for each data) using CellMarker DB. (a) tumor data with MarkerCount, (b) tumor data with Garnett, (c) blood with MarkerCount and (d) blood with SCINA. For identification, we used cell types as provided in marker DB, either broad or specific. After cell types are identified, we renamed the cell types to the respective broad type to evaluate performances. To give insight into the performance for specific cell types, we also showed in (e) and (f) the Sankey diagrams for PBMC 68K, for which the specific cell types were annotated. 

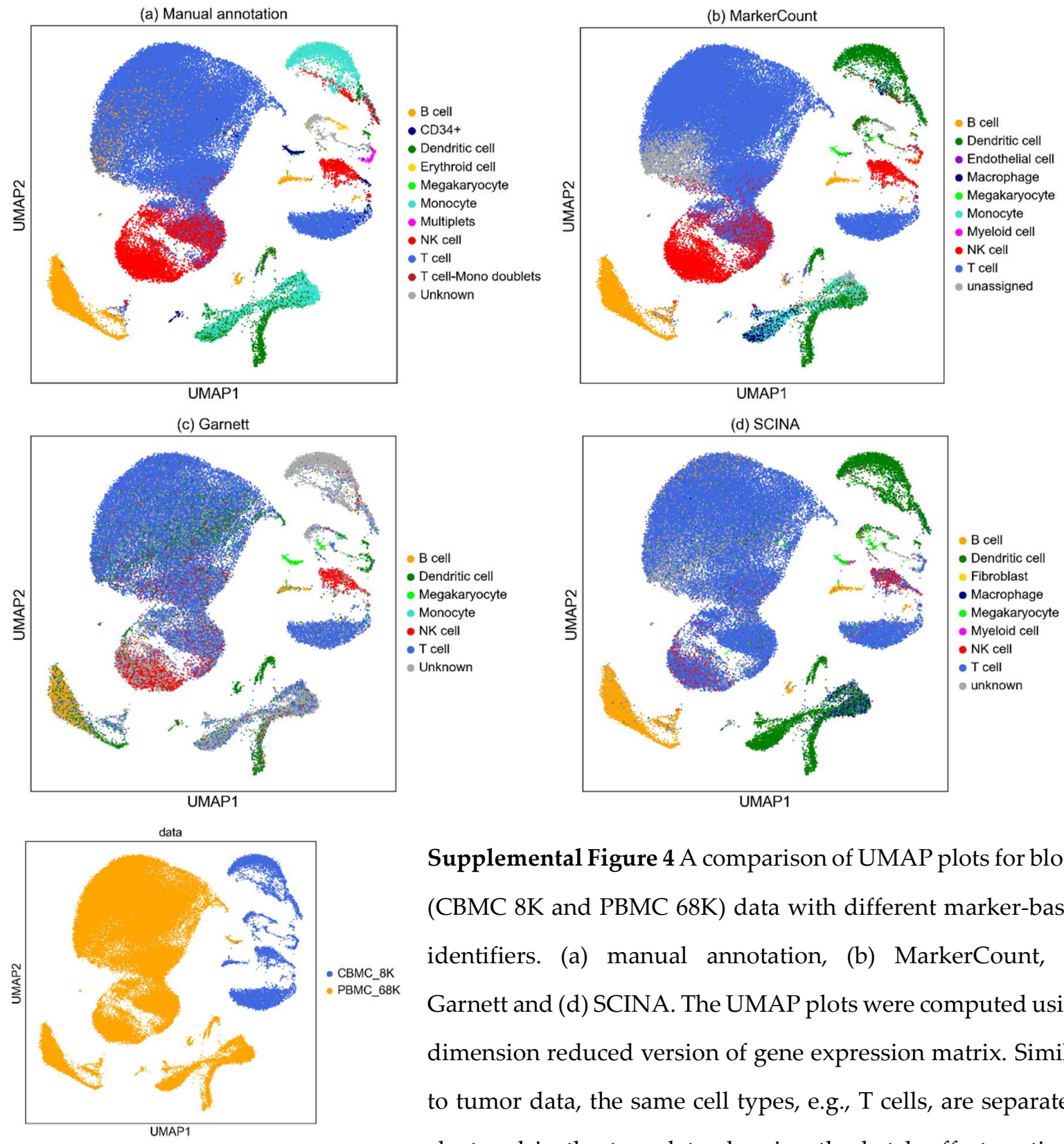

Supplemental Figure 4 A comparison of UMAP plots for blood (CBMC 8K and PBMC 68K) data with different marker-based identifiers. (a) manual annotation, (b) MarkerCount, (c) Garnett and (d) SCINA. The UMAP plots were computed using dimension reduced version of gene expression matrix. Similar to tumor data, the same cell types, e.g., T cells, are separately clustered in the two data showing the batch effect or tissue dependency. 
(a) Pancreas_2K with Pancreas_8K used as reference to find markers

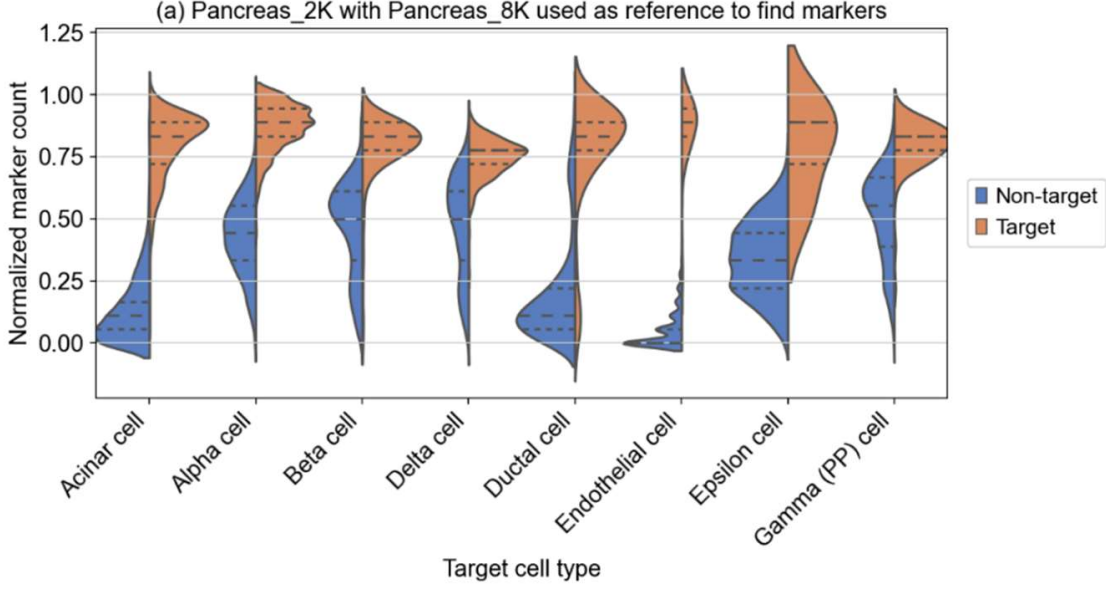

(b) CBMC_8K with PBMC_68K used as reference to find markers
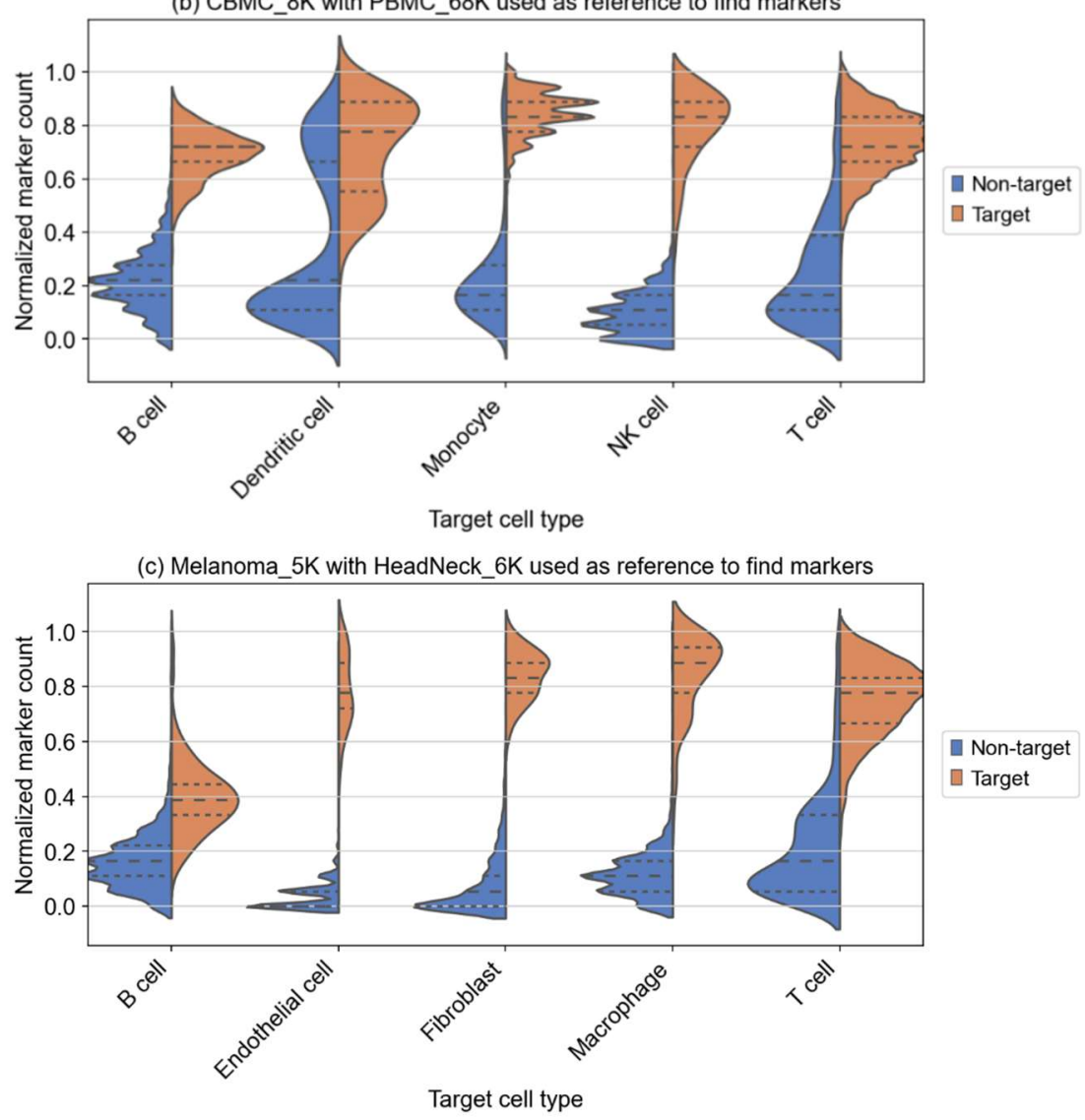

Supplemental Figure 5 Comparisons of the two distributions of $C_{m}$ and $\bar{C}_{m}$ for some cell types in (a) pancreas data, (b) blood data (CBMC 8K and PBMC68K) and (c) tumor (HeadNeck 6K and melanoma 5K) data. For many cell types, the target and non-target are largely separable using only the normalized marker counts, while for some other types, for example, Beta, Delta, Epsilon and Gamma cells in pancreas, dendritic cells in blood and B cells in tumor data, identification using only the normalized marker counts will have considerable errors. Taking such problem into account, MarkerCount set rejection threshold for the normalized marker counts and use cluster-basis cell type reassignment utilizing the similarity within a cluster. One more thing to note is that, in blood data, a large portion of non-dendritic cells have normalized marker counts comparable with those of dendritic cell. These cells were mostly monocytes (based on manual annotation) which means they have expressed marker genes both of monocytes and dendritic cells. 